\title{
Influence of Cavity Decay on Phase Distribution and Rabi Flopping in Cavity QED
}

\author{
Arpita Ghosh • P.K. Das
}

Received: 6 September 2007 / Accepted: 8 November 2007 / Published online: 21 November 2007

(C) The Author(s) 2007

\begin{abstract}
The quantum mechanical phase distribution and the quantum oscillations of population are studied for a cavity field togetherwith a driven classical field. The atom is sent through the cavity and driven by a classical field. Also the time evolution of the system including decay is obtained. The graph showing the Rabi oscillations for this system is changed into a bit pattern from the system without decay. The phase probability function is also affected due to the presence of cavity decay.
\end{abstract}

Keywords Cavity decay $\cdot$ Phase distribution $\cdot$ Rabi flopping

\section{Introduction}

It is well-known that electromagnetic field at resonance with a two-level quantum mechanical system gives rise to coherent oscillations in the populations of the two levels known as Rabi oscillation [1]. Rabi-flopping concept stands for a basic model on which virtually all subsequent theories and experiments on coherent optical pulses interacting with twolevel atoms [2] depend. Again, quantum bits (qubits) are the fundamental building blocks of quantum information processors, such as quantum computers. A qubit which can be manipulated with a high-speed voltage pulse controls the energy and decoherence of the system. As the ability to produce Rabi oscillations in a given system indicates whether that system is a candidate for more elaborate coherent manipulation or not, we are interested to study the quantum collapse and revival phenomena in details.

Recently, Deng et al. [3] proposed a scheme for generating the $n$-qubit $W$ state in the presence of cavity decay by resonant interaction between atoms and the cavity with initial single-photon cavity field and all atoms initially in the ground state. We take into account this

\footnotetext{
A. Ghosh · P.K. Das ( $\varangle)$

Physics and Applied Mathematics Unit, Indian Statistical Institute, 203 B. T. Road,

Kolkata 700108, India

e-mail: daspk@isical.ac.in
}

A. Ghosh

e-mail: arpitag_r@isical.ac.in 
idea and apply it to find out the influence of cavity decay on different physical properties of an atom-field system. Theoretical studies of Rabi oscillations have already been made [4-6]. But they did not take into account the effect of cavity decay. Conditioned on no photon leakage from the cavity, we analyze the influence of decay to the Rabi oscillation.

Here a two-level atom is sent through the cavity and driven by a weak classical field. The atom interacts with the cavity field. The frequency for atomic transition is to be tuned in resonance with the frequency of the classical field and it can be observed that Rabi oscillations can occur subject to some constraints. Also we have investigated the effect of cavity decay onto the Rabi flopping.

Pegg and Barnett (1989) constructed a Hermitian phase operator [7], the eigenstates of which, in an appropriate limit, generate the correct phase statistics for arbitrary states. The phase distribution provides a useful insight into the structure of quantum states. Though several authors investigated the phase properties of different states but less attention has been paid to the influence of cavity decay on phase distribution. We discuss here this interesting feature for the atom-field interaction for both without and with decay of the field according to Peng and Li's approach [8]. We observe that the cavity decay term leads to some changes to the probability density function.

The paper is organized as follows. In Sect. 2, we discuss the entanglement without decay. In Sect. 3, we study the same with decay of the field. And finally we give conclusion and discussion in Sect. 4.

\section{Entanglement without Decay}

We first consider a two-level atom interacting with a single mode cavity and driven by a weak classical field in the absence of any decay. The Hamiltonian (assuming $\hbar=1$ ) describing the atom-field interaction in the rotating-wave approximation is [9]

$$
H=\omega_{0} S_{z}+\omega_{a} a^{\dagger} a+g\left(a^{\dagger} S^{-}+a S^{+}\right)+\epsilon\left(S^{+} e^{-i \omega t}+S^{-} e^{i \omega t}\right)
$$

where $a^{\dagger}$ and $a$ are, respectively, the creation and annihilation operators for the cavity field, $S_{z}, S^{+}, S^{-}$are the inversion, raising and lowering operators for the atom respectively, $g$ is the coupling constant between the atom and the cavity field, $\epsilon$ is the coupling constant between the atom and the classical field. $\omega_{0}, \omega_{a}$ and $\omega$ are the frequencies for atomic transition, cavity mode and classical field respectively.

If the reference frame of the system is rotated with respect to the driving field frequency $\omega$, the Hamiltonian is given by

$$
H_{r}=\Delta S_{z}+\delta a^{\dagger} a+g\left(a^{\dagger} S^{-}+a S^{\dagger}\right)+\epsilon\left(S^{+}+S^{-}\right)
$$

where $\Delta=\omega_{0}-\omega, \delta=\omega_{a}-\omega$. For the sake of simplicity, taking $\Delta=0$, the Hamiltonian in the interaction picture is

$$
\begin{aligned}
H_{I} & =e^{i H_{r_{0}} t} H_{r_{1}} e^{-i H_{r_{0}} t} \\
& =\frac{1}{2} g\left[|+\rangle\langle+|-|-\rangle\left\langle-\left|+e^{i 2 \epsilon t}\right|+\right\rangle\left\langle-\left|-e^{-i 2 \epsilon t}\right|-\right\rangle\langle+|\right] a e^{-i \delta t}+\text { H.c. }
\end{aligned}
$$

where

$$
H_{r_{0}}=\delta a^{\dagger} a+\epsilon\left(S^{+}+S^{-}\right)
$$


and

$$
H_{r_{1}}=g\left(a^{\dagger} S^{-}+a S^{\dagger}\right)
$$

where the dressed states $| \pm\rangle=\frac{1}{\sqrt{2}}(|g\rangle \pm|e\rangle)$ are eigenstates of $\left(S^{+}+S^{-}\right)$with eigenvalues \pm 1 . In the strong driving regime $\epsilon \gg\{\delta, g\}$. So the terms that oscillate with high frequencies can be eliminated. The effective Hamiltonian under the rotating-wave approximation is [10]

$$
\begin{aligned}
H_{\mathrm{eff}} & =\frac{1}{2} g(|+\rangle\langle+|-|-\rangle\langle-|)\left(a e^{-i \delta t}+a^{\dagger} e^{i \delta t}\right) \\
& =\frac{1}{2} g\left(a S^{+} e^{-i \delta t}+a^{\dagger} S^{-} e^{i \delta t}\right)
\end{aligned}
$$

We are now going to solve the Schrödinger equation of motion

$$
i \frac{\partial|\psi(t)\rangle}{\partial t}=H_{\mathrm{eff}}|\psi(t)\rangle
$$

for any arbitrary state $|\psi\rangle[11]$. The equations of motion for the probability amplitudes $c_{e, n}$ and $c_{g, n+1}$ are

$$
\left.\begin{array}{rl}
\dot{c}_{e, n} & =-\frac{i g}{2} \sqrt{n+1} e^{-i \delta t} c_{g, n+1} \\
c_{g, n+1} & =-\frac{i g}{2} \sqrt{n+1} e^{i \delta t} c_{e, n}
\end{array}\right\}
$$

These equations can be solved exactly subject to certain initial conditions. If the atom enters the cavity in its excited state $|e\rangle$ then

$$
c_{e, n}(0)=c_{n}(0) \quad \text { and } \quad c_{g, n+1}(0)=0
$$

where $c_{n}(0)$ is the probability amplitude for the field alone. With these initial conditions, a general solution for the above system is

$$
c_{e, n}(t)=c_{n}(0)\left\{\cos \frac{\Omega_{n} t}{2}+\frac{i \delta}{\Omega_{n}} \sin \frac{\Omega_{n} t}{2}\right\} e^{\frac{-i \delta t}{2}}
$$

and

$$
c_{g, n+1}(t)=-c_{n}(0) \frac{i g \sqrt{n+1}}{\Omega_{n}} \sin \frac{\Omega_{n} t}{2} e^{\frac{i \delta t}{2}}
$$

where $\Omega_{n}^{2}=\delta^{2}+g^{2}(n+1)$.

\subsection{Phase Distribution}

Here we investigate the phase-probability distribution of the radiation field in the model we discussed above using the formalism of Pegg and Barnett.

For the exact resonance case, (7) and (8) change into

$$
\begin{aligned}
c_{e, n}(t) & =\frac{c_{n}(0)}{2}\left\{e^{-i \gamma_{n} t}+e^{i \gamma_{n} t}\right\} \\
c_{g, n+1}(t) & =\frac{c_{n}(0)}{2}\left\{e^{-i \gamma_{n} t}-e^{i \gamma_{n} t}\right\}
\end{aligned}
$$


where $\gamma_{n}^{2}=\frac{g^{2}}{4}(n+1)$.

With the help of (9) and (10), the evolution of the state vector is described in the interaction picture. We change it into the Schrödinger picture as

$$
|\psi(t)\rangle=\sum_{n=0}^{\infty}\left[c_{e, n}(t)|e, n\rangle+c_{g, n+1}(t)|g, n+1\rangle\right] \exp \left\{-i(n+1 / 2) \omega_{a} t\right\}
$$

The Pegg-Barnett phase operator operates on an $(s+1)$ dimensional subspace spanned by the number states $|0\rangle,|1\rangle, \ldots,|s\rangle$. The value of s can be made arbitrarily large. A complete orthonormal basis of the $(s+1)$ phase state is defined on the finite subspace by

$$
\left|\theta_{m}\right\rangle=(s+1)^{-1 / 2} \sum_{n=0}^{s} \exp \left(\operatorname{in} \theta_{m}\right)|n\rangle
$$

with

$$
\theta_{m}=\theta_{0}+2 m \pi /(s+1), \quad m=0,1,2, \ldots, s
$$

The value of $\theta_{0}$ is arbitrary. These states form a complete orthonormal set on the truncated $(s+1)$ dimensional Hilbert space. These states are the eigenstates of the Pegg-Barnett Hermitian phase operator

$$
\Phi=\sum_{m=0}^{s} \theta_{m}\left|\theta_{m}\right\rangle\left\langle\theta_{m}\right|
$$

One should take the limit $s \rightarrow \infty$ and recover an Hermitian phase operator on the full Hilbert space. Thus the state vector $|\psi(t)\rangle$ of the atom-field coupling system is spanned as

$$
|\psi(t)\rangle=\sum_{m=0}^{s}\left[\left\langle e, \theta_{m} \mid \psi(t)\right\rangle\left|e, \theta_{m}\right\rangle+\left\langle g, \theta_{m} \mid \psi(t)\right\rangle\left|g, \theta_{m}\right\rangle\right]
$$

We assume that the radiation field is initially in coherent state. Then

$$
c_{n}(0)=\frac{\alpha^{n} e^{-\bar{n} / 2}}{\sqrt{n !}}
$$

where

$$
\alpha=(\bar{n})^{1 / 2} e^{i \theta_{\alpha}}
$$

$\bar{n}$ is the mean photon number and $\theta_{\alpha}$ is the phase angle of $\alpha$. For large $|\alpha|^{2}$, we may use the Gaussian approximation for a Poisson distribution

$$
c_{n}(0) \approx(2 \pi \bar{n})^{-1 / 4} \exp \left[-\frac{(n-|\alpha|)^{2}}{4 \bar{n}}\right] e^{i n \theta_{\alpha}}
$$

Recalling the saddle-point approximation method, we get

$$
\gamma_{n}=\sqrt{\frac{g^{2}}{4}(n+1)} \approx \frac{g n}{4 \sqrt{\bar{n}}}
$$


We now use (15) and (16) to write the phase-probability distribution as

$$
\begin{aligned}
P\left(\theta_{m}, t\right)= & \left|\left\langle e, \theta_{m} \mid \psi(t)\right\rangle\right|^{2}+\left|\left\langle g, \theta_{m} \mid \psi(t)\right\rangle\right|^{2} \\
= & \frac{1}{8} \frac{2 \pi}{(s+1)}\left(\frac{4 \bar{n}}{2 \pi}\right)^{1 / 2}\left[\exp \left\{-2 \bar{n}\left(\theta_{\alpha}-\theta_{m}-\omega_{a} t-\frac{g t}{4 \sqrt{\bar{n}}}\right)^{2}\right\}\right. \\
& \left.+\exp \left\{-2 \bar{n}\left(\theta_{\alpha}-\theta_{m}-\omega_{a} t+\frac{g t}{4 \sqrt{\bar{n}}}\right)^{2}\right\}\right]
\end{aligned}
$$

When $s \rightarrow \infty, \theta_{m}$ is a continued variation.

The phase-probability distribution is normalized as

$$
\int P(\theta, t) \frac{4(s+1)}{2 \pi} d \theta=1
$$

where $\frac{4(s+1)}{2 \pi}$ is the density of the states.

If we consider $\frac{g}{4 \sqrt{\bar{n}}} \ll \omega_{a}$ then we can neglect the term $\frac{g}{4 \sqrt{\bar{n}}}$ compared to $\omega_{a}$. Using (17), we obtain the phase fluctuation as in [8]

$$
\langle\Delta \Phi\rangle^{2}=\left\langle\Phi^{2}\right\rangle-\langle\Phi\rangle^{2}=\frac{1}{4 \bar{n}}
$$

If the photon number distribution is Poissonian we have

$$
\langle\Delta N\rangle^{2}=\bar{n}
$$

so that the uncertainty product is

$$
\langle\Delta \Phi\rangle^{2}\langle\Delta N\rangle^{2}=\frac{1}{4}
$$

i.e. neglecting the influence of the atomic Rabi oscillation on the phase-probability distribution we can show that the field which is initially in a coherent state remains coherent.

\subsection{Population Inversion}

The population inversion $W(t)$ which is very useful in probing the effect of the quantum nature of the driving field is related to the probability amplitudes $c_{e, n}(t)$ and $c_{g, n}(t)$ by the expression

$$
\begin{aligned}
W(t) & =\sum_{n}\left[\left|c_{e, n}(t)\right|^{2}-\left|c_{g, n}(t)\right|^{2}\right] \\
& =\sum_{n} \rho_{n n}(0)\left[\frac{\delta^{2}}{\Omega_{n}^{2}}+\frac{g^{2}(n+1)}{\Omega_{n}^{2}} \cos \Omega_{n} t\right]
\end{aligned}
$$

where $\rho_{n n}(0)=\left|c_{n}(0)\right|^{2}$ is the probability that there are $n$ photons present in the field at time $t=0$. For an initial coherent state, $\rho_{n n}(0)=\frac{\bar{n}^{n} e^{-\bar{n}}}{n !}$ where $\bar{n}$ is the mean photon number, $\alpha=(\bar{n})^{1 / 2}$.

In Fig. 1, $W(t)$ is plotted as a function of time for an initial coherent state. Each term in the summation (18) represents Rabi oscillations for a definite value of $n$. At the initial time, 


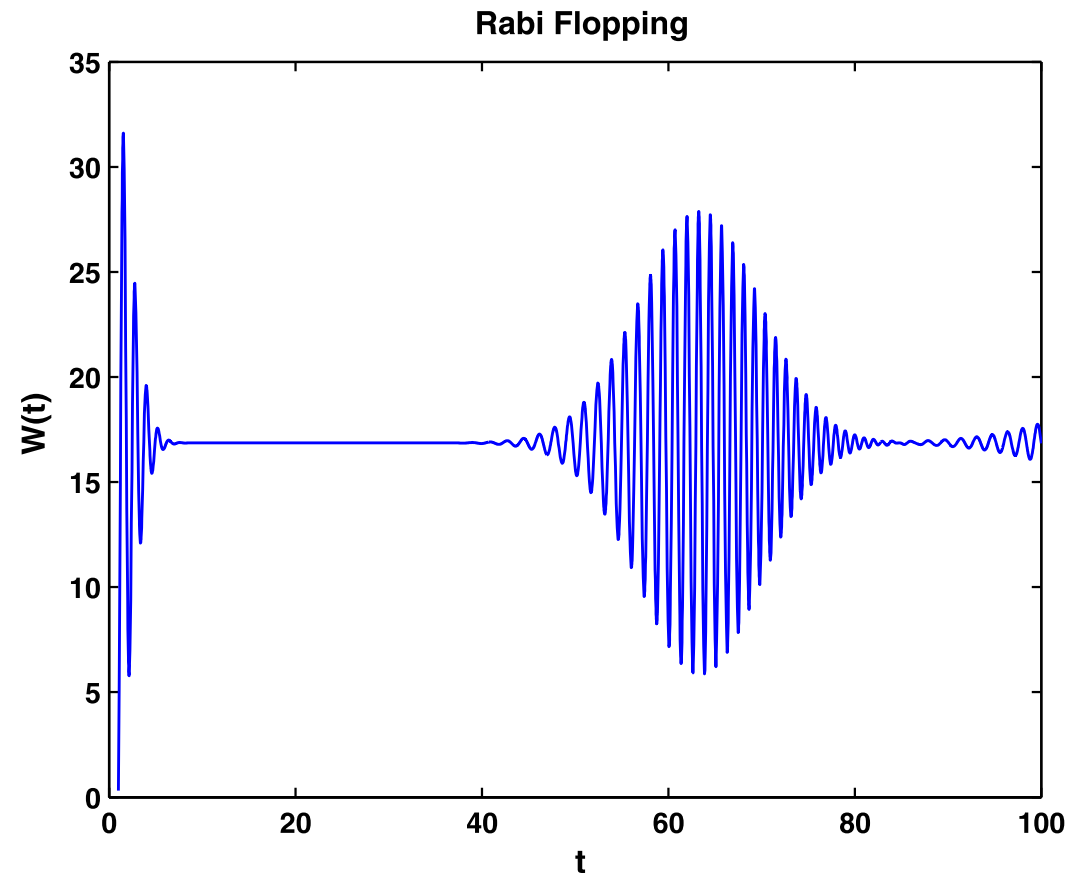

Fig. 1 Time-dependence of the population inversion $W(t)$ for an initially coherent state with $\bar{n}=25, \delta=0$, $g=1, n$ ranging from 0 to 100

$t=0$, the atom is prepared in a definite state and therefore all the terms in the summation are correlated. As time increases the Rabi oscillations associated with different excitations have different frequencies and therefore become uncorrelated leading to a collapse of inversion. As time increases the Rabi oscillation collapses to zero and revival occurs. This behavior of collapse and revival of inversion is continued with increasing time, with the amplitude of Rabi oscillations decreasing and the time duration in which revival takes place increasing and ultimately overlapping with the earlier revival.

\section{Entanglement with Decay}

Now we introduce the cavity decay. As long as there is no photon decay from the cavity, the evolution of the system is governed by the non-hermitian Hamiltonian [3]

$$
H^{\prime}=H-\frac{i k}{2} a^{\dagger} a
$$

where $k$ is the cavity decay rate.

In strong driving regime, the effective Hamiltonian as in Sect. 2 is given by

$$
H_{\mathrm{eff}}^{\prime}=\frac{1}{2} g\left(a S^{+} e^{-i \delta^{\prime} t}+a^{\dagger} S^{-} e^{i \delta^{\prime} t}\right)
$$

where $\delta^{\prime}=\delta-i k / 2$. 
Solving the equation of motion for any arbitrary state

$$
\left|\psi^{\prime}\right\rangle=\sum_{n=0}^{\infty}\left[d_{e, n}|e, n\rangle+d_{g, n}|g, n\rangle\right]
$$

and simplifying we get the equation for $d_{e, n}$ as

$$
\ddot{d}_{e, n}+i \delta^{\prime} \dot{d}_{e, n}+\frac{g^{2}(n+1)}{4} d_{e, n}=0
$$

This is a second order equation with complex coefficients. Assuming that the atom is initially in the excited state we obtain

$$
d_{e, n}(t)=\frac{d_{n}(0)}{2}\left\{D_{1} e^{v t}+D_{2} e^{-v t}\right\} e^{\frac{-i \delta^{\prime} t}{2}}
$$

and

$$
d_{g, n+1}(t)=-\frac{i g \sqrt{n+1}}{2} \frac{d_{n}(0)}{2}\left\{\frac{D_{1} e^{\left(v+\frac{i \delta^{\prime}}{2}\right) t}}{\left(v+\frac{i \delta^{\prime}}{2}\right)}-\frac{D_{2} e^{-\left(v-\frac{i \delta^{\prime}}{2} t\right)}}{\left(v-\frac{i \delta^{\prime}}{2}\right)}\right\}
$$

where

$$
\begin{aligned}
D_{1} & =1+\frac{i \delta^{\prime}}{u} e^{-i \theta / 2} \\
D_{2} & =1-\frac{i \delta^{\prime}}{u} e^{-i \theta / 2} \\
v & =\frac{u e^{i \theta / 2}}{2} \\
u & =\left(\lambda_{n}^{2}+\delta^{2} k^{2}\right)^{1 / 4} \\
\theta & =\tan ^{-1}\left(\frac{\delta k}{\lambda_{n}}\right) \\
\lambda_{n} & =\frac{k^{2}}{4}-\Omega_{n}{ }^{2}
\end{aligned}
$$

For exact resonance case we get finally

$$
d_{e, n}(t)=e^{-\frac{k t}{4}} d_{n}(0)\left[\cosh \left(\frac{\sqrt{\lambda_{n}} t}{2}\right)+\frac{k}{2 \sqrt{\lambda_{n}}} \sinh \left(\frac{\sqrt{\lambda_{n}} t}{2}\right)\right]
$$

and

$$
d_{g, n+1}(t)=-i g \sqrt{n+1} \frac{d_{n}(0)}{\sqrt{\lambda_{n}}} e^{\frac{k t}{4}} \sinh \left(\frac{\sqrt{\lambda_{n}} t}{2}\right)
$$

\subsection{Phase Distribution}

Using Pegg and Barnett formalism we now investigate the phase-probability distribution of the radiation field in the model discussed above.

For exact resonance case we use the approximation

$$
\sqrt{\lambda_{n}}=i g \sqrt{n+1} \sqrt{1-\frac{k^{2}}{4 g^{2}(n+1)}} \approx i g \frac{n}{2 \sqrt{\bar{n}}} .
$$


With this approximation, (24) and (25) change into

$$
d_{e, n}(t)=e^{-k t / 4} \frac{d_{n}(0)}{2}\left[\exp \left(-i \frac{g n t}{4 \sqrt{\bar{n}}}\right)+\exp \left(i \frac{g n t}{4 \sqrt{\bar{n}}}\right)\right]
$$

and

$$
d_{g, n+1}(t)=e^{k t / 4} \frac{d_{n}(0)}{2}\left[\exp \left(-i \frac{g n t}{4 \sqrt{\bar{n}}}\right)-\exp \left(i \frac{g n t}{4 \sqrt{\bar{n}}}\right)\right]
$$

Assuming that the radiation field is initially in a coherent state and using the Gaussian approximation as in (15) we finally obtain the phase probability as

$$
\begin{aligned}
P\left(\theta_{m}, t\right)= & \left|\left\langle e, \theta_{m} \mid \psi(t)\right\rangle\right|^{2}+\left|\left\langle g, \theta_{m} \mid \psi(t)\right\rangle\right|^{2} \\
= & \frac{\bar{n} \pi}{s+1} \frac{(2 \pi \bar{n})^{-1 / 2}}{4}\left[e^{-2 \bar{n}\left(A^{2}-\frac{k^{2} t^{2}}{4}\right)}\left(e^{-k t / 2}+e^{k t / 2}\right)+e^{-2 \bar{n}\left(B^{2}-\frac{k^{2} t^{2}}{4}\right)}\left(e^{-k t / 2}+e^{k t / 2}\right)\right. \\
& \left.-2 e^{-\bar{n}\left(A^{2}+B^{2}-\frac{k^{2} t^{2}}{2}\right)} \cos \bar{n}(A-B) k t\left(e^{k t / 2}-e^{-k t / 2}\right)\right] \\
= & \frac{1}{8} \frac{2 \pi}{(s+1)}\left(\frac{4 \bar{n}}{2 \pi}\right)^{1 / 2}\left\{\left[e^{-2 \bar{n}\left(A^{2}-\frac{k^{2} t^{2}}{4}\right)}+e^{-2 \bar{n}\left(B^{2}-\frac{k^{2} t^{2}}{4}\right)}\right] \cosh \left(\frac{k t}{2}\right)\right. \\
& \left.-2 e^{-\bar{n}\left(A^{2}+B^{2}-\frac{k^{2} t^{2}}{2}\right)} \cos \bar{n}(A-B) k t \sinh \left(\frac{k t}{2}\right)\right\}
\end{aligned}
$$

where

$$
\begin{aligned}
& A=\theta_{\alpha}-\theta_{m}-\omega_{a} t-\frac{g t}{4 \sqrt{\bar{n}}} \\
& B=\theta_{\alpha}-\theta_{m}-\omega_{a} t+\frac{g t}{4 \sqrt{\bar{n}}}
\end{aligned}
$$

If we choose $\frac{g}{4 \sqrt{\bar{n}}} \ll \omega_{a}$ then we can neglect the term $\frac{g}{4 \sqrt{\bar{n}}}$ compared to $\omega_{a}$. Hence

$$
P\left(\theta_{m}, t\right)=\frac{1}{4} \frac{2 \pi}{(s+1)}\left(\frac{4 \bar{n}}{2 \pi}\right)^{1 / 2} e^{-2 \bar{n}\left(\theta_{\alpha}-\theta_{m}-\omega_{a} t\right)^{2}} e^{\frac{\bar{n} k t}{2}\left(k t-\frac{1}{n}\right)}
$$

Proceeding as in Sect. 2, we obtain that for presence of cavity decay, the phase fluctuations are changed and the field, which is initially in a coherent state, does not keep the phasenumber product.

If further we take the interaction time to satisfy $t=\frac{1}{k \bar{n}}$ then corresponding success probability is

$$
P\left(\theta_{m}, t\right)=\frac{1}{4} \frac{2 \pi}{(s+1)}\left(\frac{4 \bar{n}}{2 \pi}\right)^{1 / 2} e^{-2 \bar{n}\left(\theta_{\alpha}-\theta_{m}-\omega_{a} t\right)^{2}}
$$

and we have

$$
\int \frac{4(s+1)}{2 \pi} P(\theta, t) d \theta=1
$$

Thus we see that the phase probability increases with the increase of $k$ in general but for a certain interaction time, the cavity decay rate $k$ has no effect on the distribution. 


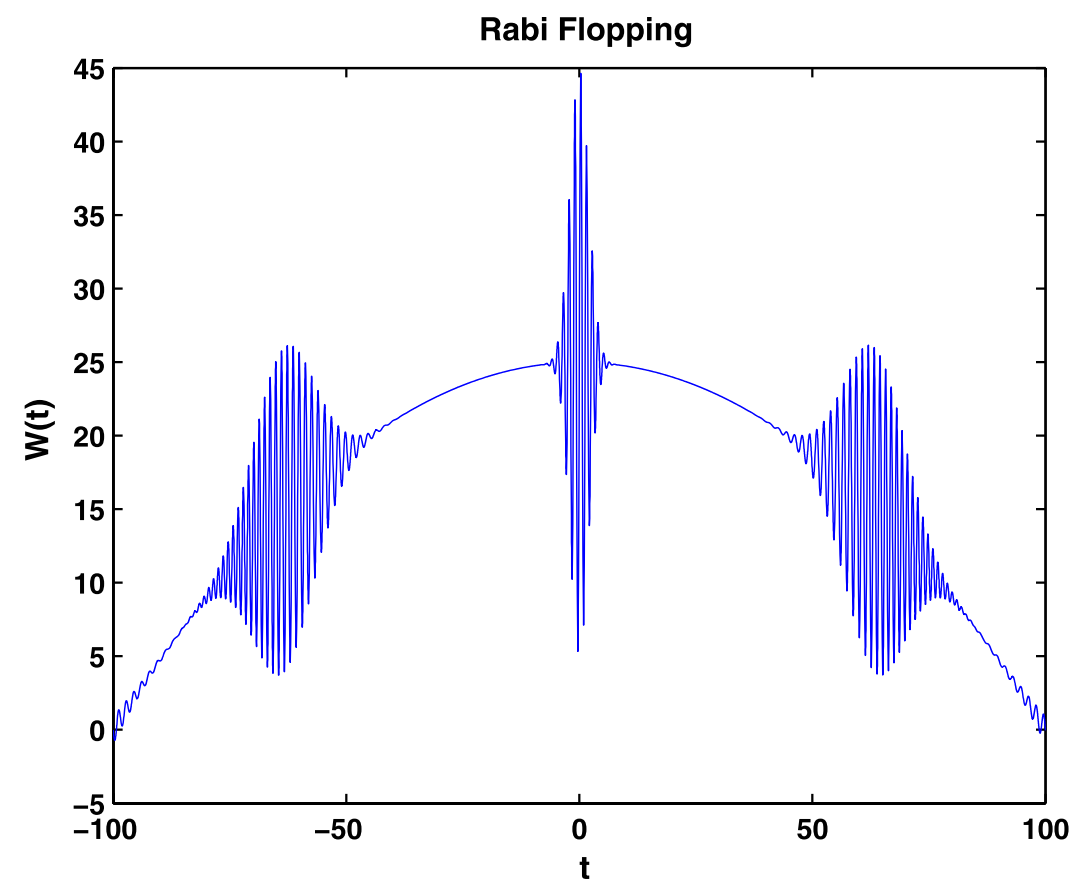

Fig. 2 The population inversion $W(t)$ versus the interaction time for an initially coherent state with $\bar{n}=25$, $\delta=0, g=1, k=0.0001, n$ ranging from 0 to 100

\subsection{Population Inversion}

We are now interested in the atomic population inversion considering the decay of the field. Under the condition of exact resonance, a closed expression for $W(t)$ can be calculated as

$$
\begin{aligned}
W(t)= & \sum_{n} \rho_{n n}(0)\left[e^{-\frac{k t}{2}}\left\{\cosh \left(\frac{\sqrt{\lambda_{n}} t}{2}\right)+\frac{k}{2 \sqrt{\lambda_{n}}} \sinh \left(\frac{\sqrt{\lambda_{n}} t}{2}\right)\right\}^{2}\right. \\
& \left.-\frac{g^{2}(n+1)}{\lambda_{n}} e^{\frac{k t}{2}} \sinh ^{2}\left(\frac{\sqrt{\lambda_{n}} t}{2}\right)\right]
\end{aligned}
$$

where $\lambda_{n}$ is given by (23). Each term of (33) represents Rabi oscillations for a definite value of $n$. The photon distribution function $\rho_{n n}(0)$ stands for the relative weight for each $n$.

Figure 2 shows the comparison between the population inversion $W(t)$, considering the decay effect, and the time period. This figure exhibits the existence of periodic spontaneous collapse and revival for the above model.

\section{Discussion and Conclusion}

We have studied phase distribution of the radiation field through the Pegg-Barnett phase formalism. We have investigated the effect of cavity decay on a two-level atom interacting with a single-mode cavity and driven by a classical field. The general expression shows the dependence of the phase probability on the cavity decay but for a particular interaction time, 

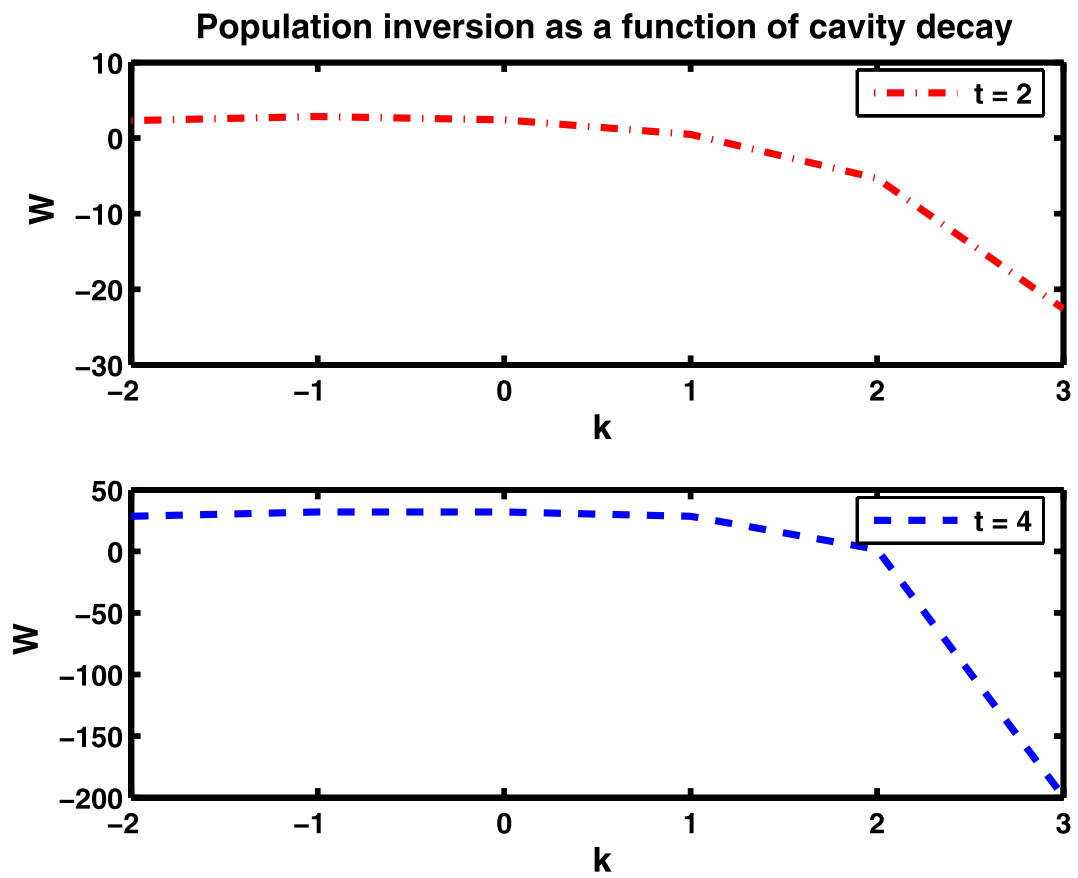

Fig. 3 The population inversion $W$ versus the cavity decay rate $k$ for an initially coherent state with $\bar{n}=25$, $\delta=0, g=1, n$ ranging from 0 to 100

this phase probability is tuned with the phase probability of without decay case. Another point we would like to mention that, in absence of cavity decay, the field which is initially in a coherent state, retains coherence with time development if one neglects the influence of the atomic Rabi oscillations on the phase probability distribution. But the presence of cavity decay leads to phase fluctuation of the radiation field i.e. the coherent field cannot keep its phase-number-minimum-uncertainty product.

While studying the population inversion we have observed that the decay term makes a contribution to the population inversion, thus changes the ideal Rabi oscillation into a bit pattern. We have numerically calculated equation (33) for some particular values and shown the dependence of population inversion on cavity decay rate $\mathrm{k}$ in different cases in Fig. 3. For a certain time, the population inversion decreases, slowly when $k \leq 2$ and rapidly after $k=2$, with increase of $k$, implying that we spend more time to achieve the inversion in the decay case than in the ideal case. The plotted graph clearly demonstrates that the curve corresponding to $t=4$ drops more sharply than that of $t=2$. Therefore the cavity decay has larger impact on the curve as time increases.

In conclusion, our approach presents analytical expressions for the influence from cavity decay on phase distribution and population inversion. Our study would be helpful for experimental work dealing with problems of cavity decay.

Acknowledgement This work is supported by the Council of Scientific and Industrial Research under Grant No. 09/093(0092)/2006-EMR-I.

Open Access This article is distributed under the terms of the Creative Commons Attribution Noncommercial License which permits any noncommercial use, distribution, and reproduction in any medium, provided the original author(s) and source are credited. 


\section{References}

1. Rabi, I.I.: Phys. Rev. 51, 652 (1937)

2. Allen, L., Eberly, J.H.: Optical Resonance and Two-Level Atoms. Dover, New York (1987)

3. Deng, Z.J., Feng, M., Gao, K.L.: Phys. Rev. A 73, 014302 (2006)

4. Eberly, J.H., Narozhny, N.B., Sanchez-Mondragon, J.J.: Phys. Rev. Lett. 44(20), 1323 (1980)

5. Wu, Z.-J., Zhu, K.-D., Yuan, X.-Z., Jiang, Y.-W., Yao, M.: Phys. Lett. A 347, 251 (2005)

6. Batista, A.A., Citrin, D.S.: Phys. Rev. Lett. 92(12), 127404 (2004)

7. Walls, D.F., Milburn, G.J.: Quantum Optics. Springer, Berlin (1994)

8. Peng, J.S., Li, G.X.: Phys. Rev. A 45(5), 3289 (1992)

9. Zheng, S.-B.: Phys. Rev. A 74, 043803 (2006)

10. Chen, C.-Y., Feng, M., Gao, K.-L.: Phys. Rev. A 73, 034305 (2006)

11. Scully, M.O., Suhail Zubairy, M.: Quantum Optics. Cambridge University Press, Cambridge (1997) 